\title{
S-resistin, a non secretable resistin isoform, impairs the insulin signalling pathway in 3T3-L1 adipocytes
}

María Rodríguez ${ }^{\mathrm{a}}$, Eduardo Moltóa ${ }^{\mathrm{a}}$ Lidia Aguado $^{\mathrm{a}}$, Nilda Gallardo ${ }^{\mathrm{b}}$, Antonio Andrés $^{\mathrm{b}}$ and Carmen Arribas $^{\mathrm{a} *}$

aÁrea de Bioquímica. Facultad de Ciencias Ambientales y Bioquímica, Centro Regional de Investigaciones Biomédicas (CRIB), Universidad de Castilla-La Mancha, Av. Carlos III s/n 45071Toledo, Spain.

bÁrea de Bioquímica. Facultad de Ciencias y Tecnologías Químicas, Centro Regional de Investigaciones Biomédicas (CRIB), Universidad de Castilla-La Mancha, Av Camilo José Cela s/n, 13071-Ciudad Real, Spain.

*Corresponding author: Carmen Arribas; Phone: 34-925268800 Ext: 5411. Fax: 34-925268840. E-mail: carmen.arribas@uclm.es 


\section{ABSTRACT}

S-resistin is a non-secretable resistin spliced variant, which is expressed mainly in the white adipose tissue from Wistar rats. Previous results confirmed that 3T3-L1 cells expressing s-resistin (3T3-L1-sres) showed an inflammatory response and exhibited a decrease in glucose transport, both basal and insulin-stimulated. Here we present evidences demonstrating for the first time that s-resistin, like resistin, blocks insulin signalling pathway by inhibiting insulin receptor, insulin receptor substrate 1, protein kinase B/Akt and the mammalian target of rapamycin phosphorylation, and increasing the suppressor of cytokine signalling 3 levels being the later probably due to augmented of leptin expression. Thus, our data suggest that s-resistin could act by a still unknown intracrine pathway as an intracellular sensor, regulating the adipocyte insulin sensitivity.

Keywords: Resistin, s-resistin, insulin-resistance, type 2-diabetes, leptin-resistance, inflammation. 


\section{INTRODUCTION}

The control of body weight, glucose and lipid homeostasis is exerted by a complex network composed by peripheral tissues, neuronal circuits of the central nervous system (CNS), as well as by several hormones such as leptin, insulin or resistin [1]. The adipose tissue secretes cytokines and growth factors, named adipokines, which act in a paracrine-endocrine way on peripheral tissues modulating their response to different nutritional factors. Furthermore, adipocytes express receptors for most of these factors, constituting by itself autocrine targets for these signals [2].

Since its characterization in rodents, the adipokine resistin has been implicated in insulin resistance, obesity and type 2 diabetes development [3, 4]. Initial results showed that in vivo administration of recombinant resistin impairs glucose tolerance and insulin action in normal mice, whereas anti-resistin antibody administration enhances insulin sensitivity of insulin-resistant and obese mice [3]. Moreover, according to Banerjee et al. [5], mice lacking resistin display low blood glucose levels after fasting, indicating that resistin acts on peripheral tissues, disrupting insulin response at different levels. Thus, in liver, resistin promotes phosphoenolpyruvate carboxykinase (PEPCK) activity, increasing endogenous glucose production [5], whereas in adipose tissue it decreases fatty acid oxidation, increasing the levels of free fatty acid in plasma [6]. Both effects contribute to the development of insulin resistance.

In rodents, resistin has also been involved in the regulation of adipocyte differentiation through a feedback mechanism [4]. Several studies showed that overexpression of resistin in 3T3-L1 fibroblast increased the expression and secretion of proinflammatory cytokines such as tumor necrosis factor alpha $(\mathrm{TNF} \alpha)$ and interleukin 6 (IL-6) and decreased the insulin stimulated glucose uptake. These results suggest that an inflammatory pathway might mediate resistin effects impairing insulin action and adipogenesis [7, 8]. Although the resistin receptor has not been identified yet, it was described that some mouse receptors, as an isoform of decorin or the tyrosine kinase-like orphan receptor (ROR) 1, could act as functional resistin receptors and therefore, mediate some resistin functions such glucose uptake and adipogenesis $[9,10]$.

In Wistar rats, we have identified a novel isoform of resistin, named s-resistin (hhort-resistin), which is expressed mainly in the white adipose tissue. This isoform is generated by alternative splicing 
from rat resistin gene [11]. S-resistin lacks the secretion signal peptide and, in transfected cells, shows a predominantly nuclear location. Thus, the splicing machinery generates a non-secretable protein that could act as an intracrine regulator of gene expression. Interestingly, resistin and s-resistin expression is differentially regulated by fat depot, aging or nutritional status [12]. These data point out that alternative splicing plays a role in this regulation.

Previous studies with 3T3-L1 adipocytes stably expressing resistin and s-resistin, demostrated that both isoforms modified in a different way the expression pattern of key pro-adipogenic transcription factors. Likewise, both isoforms impair at different degrees the insulin-stimulated glucose transport, and promote IL-6 and plasminogen activity inhibitor type 1 (PAI-1) secretion, but only s-resistin increased the $\mathrm{TNF} \alpha$ expression and secretion in early and intermediate stages of adipocyte differentiation. These data suggest that the s-resistin variant carries out additional functions in insulin response and adipocyte differentiation [8].

Besides, several studies indicated that resistin interferes in different stages on the intracellular insulin signalling pathway. On the one hand, resistin directly influences insulin signalling increasing the suppressor of cytokine signalling 3 (SOCS-3) levels in 3T3-L1 adipocytes, and thus, inhibiting the ability of the insulin receptor (IR) to recruit and activate the insulin receptor subtrate-1 (IRS-1) [13]. In addition, resistin decreased the activated state of AMP-activated kinase (AMPK), which is involved in glucose homeostasis in liver, skeletal muscle and white adipose tissue $[6,13,14]$. However, at the moment, the effect of s-resistin on intracellular insulin signalling pathway has not been studied. Since s-resistin is an intracellular protein, it is possible that some of its effects on insulin action could be different from those described for the long resistin isoform.

In an attempt to investigate the effect of s-resistin on the intracellular insulin pathway, we compared the insulin response of adipocytes stably expressing resistin or s-resistin. Our results indicate that s-resistin impais insulin response by decreasing IR $\beta$, IRS-1 and S-473-Akt/protein kinase B phosphorylation and glucose transporter 4 (GLUT4) expression levels. Also, the changes in the expression pattern of transcription factors involved in lipid metabolism as sterol regulatory elementbinding protein 1c (SREBP-1c) and forkhead box subfamily O1a (FoxO1a), adipogenesis as mammalian target of tapamycim (mTOR) and inflammation (TNF $\alpha$ and IL-6) in adipocytes expressing s-resistin, indicates that this isoform increases the inflammatory state and promotes adipocyte insulin and leptin resistance. 


\section{MATERIALS AND METHODS}

Cell lines

Murine 3T3-L1 pre-adipocytes purchased from American Type Culture Collection, ATCC (ATTCC CL-173) was used as control and transduced cell lines 3T3-L1-res (resistin) and 3T3-L1-s-res (s-resistin) were previously obtained in our laboratory [8].

Cell culture of 3T3-L1 cells

Murine 3T3-L1 pre-adipocytes (purchased from American Type Culture Collection, ATCC) and transduced cell lines were grown in Dulbecco's modified Eagle's medium (DMEM) containing 10\% fetal bovine serum (FBS) (Gibco BRL) and $100 \mathrm{U} / \mathrm{ml}$ penicillin (Gibco) and $100 \mathrm{mg} / \mathrm{ml}$ streptomycin (Gibco) at $37{ }^{\circ} \mathrm{C}$ in a $5 \% \mathrm{CO}_{2}$ atmosphere.

Differentiation stimulation protocol

3T3-L1 fibroblast or transduced cell lines were grown and differentiated into adipocytes in p100 culture dishes, as described previously [15]. Briefly, cells were grown as described above and two days after full confluence (day 0), were differentiated by incubation during 2 days in DMEM containing 25 mM glucose, $0.8 \mathrm{mM}$ isobutylmethylxanthine (IBMX, Sigma), $1 \mu \mathrm{M}$ dexamethasone (DEX, Sigma), 174 $\mathrm{nM}$ insulin (Sigma) and 10\% FBS and then for 2 days in DMEM containing $25 \mathrm{mM}$ glucose, $174 \mathrm{nM}$ insulin and 10\% FBS. Next, cells were maintained (14 days) in the same medium without insulin until use.

Insulin incubations

Fourteen days after initiation of differentiation, cells were incubated in DMEM without FBS during 8 hours and then, they were incubated at different times with $100 \mathrm{nM}$ insulin [8]. 3T3-L1 fibroblast or transduced cell lines were grown in p60 culture dishes. The changes in the proteins of the insulin signalling pathway were analysed at 5, 10, 15 and 30 minutes in the presence and absence of insulin, whereas 4 hours was chosen to note the alterations in mRNA levels.

$\underline{\text { RNA isolation and semi-quantitative RT-PCR analysis }}$

Total RNA from 3T3-L1 cells and 3T3-L1 stably transfected cell lines was isolated using RNAEasy Mini Kit (Qiagen, Hilden, Germany) following the manufacturer's instructions. Complementary DNA (cDNA) was synthesized from $1 \mu \mathrm{g}$ of DNase-treated RNA [16].

$\underline{\text { Primers }}$ 
All the primers used in semi-quantitative and real time q-PCR were supplied by Bonsai Technologies: mTOR (sense primer, 5'-CTCTCTGACCCAGTTCGTCC-3'; antisense primer 5'AGTCTGGCTAACCACGTGGG-3'); SREBP 1c (sense primer, 5’-CTCATCAACAACCAAGACAGT3’; $\quad$ antisense primer 5'-GTGCAGGTGTCACCTTGGGT-3'); SOCS-3 (sense primer, 5' $^{\prime}$ GCGGGCACCTTTCTTATCC- 3’; antisense primer 5'-TCCCCGACTGGGTCTTGAC- 3'); Leptin (sense primer $\quad 5^{\prime}$-CACCCCATTCTGAGTTTGTCC- $\quad 3^{\prime} ; \quad$ antisense primer $\quad 5^{\prime}$ ACGCCATCCAGGCTCTC-3'); FoxO1a (sense primer 5'-CAATCTGTCCCTTCACAGCA-3'; antisense primer 5'-ACTGTTGTTGTCCATGGACG-3') and rRNA $18 \mathrm{~S}$ (sense primer 5'CGGCTACCACATCCAAGGAA-3'; antisense primer 5'-GCTGGAATTACCGCGGCT-3').

$\underline{\text { Real time q-PCR analysis }}$

Real time quantitative PCR (q-PCR) was performed by using ABI PRISM 7500 Fast Sequence Detection System instrument and software (Applied Biosystem, Foster City, CA). Relative quantification of target cDNA in each sample was performed from $10 \mathrm{ng}$ of cDNA in TaqMan One-Step real time PCR Master Mix and using Pre-Developed TaqMan Assay Reagents (PE Applied Biosystem) for: GLUT4 (Mm00436615_m1), TNFa (Mm00443258_m1), resistin (Mm00445641_m1), IL-6 (Mm00446190_m1) and 18S rRNA (Hs99999901_s1) with VIC as real time reporter was used as control to normalize gene expression. Furthermore, relative quantification of target cDNA in each sample was performed from 10 ng of cDNA in SYBR-Green One-Step real time PCR Master Mix with the primers described previously. The $\Delta \Delta \mathrm{CT}$ method was used to calculate the relative differences between experimental conditions and control groups as fold change in gene expression [17].

$\underline{\text { Protein Isolation and Western Blot analysis: }}$

Cell extracts were obtained following cell lysis in a buffer containing $10 \mathrm{mM}$ Tris $\mathrm{pH}=8 ; 120 \mathrm{mM}$ $\mathrm{NaCl}$, Triton X-100 and $1 \mathrm{mM}$ EDTA. A protease inhibitor mixture (Roche) was added before extraction. Protein concentrations were determined by the method of Bradford in accordance with the manufacturer's instructions (BioRad). The lysates were fractionated on a SDS-PAGE gel and blotted for $2 \mathrm{~h}$ at 90 V. 40 mg of protein were loaded per gel. Western Blot analysis was performed according to standard protocols. The following primary antibodies were used in this study: $\beta$-actin (Sigma, 1:1000 dilution), SOCS-3 (Santa Cruz, 1:500), pAkt S-473 (Cell Signalling, 1:500), pAkt T-308 (Cell Signalling 1:500), Akt (Cell Signaling, 1:500), pFoxO1a S-319 (Abcam 1:500), FoxO1a (Abcam 1:400), pIR $\beta$ Y-1146 (Sigma, 1:500), IR $\beta$ (Santa Cruz, 1:500), pIRS-1 Y-632(SAB Signalling, 1:500), IRS-1 (Cell Signalling, 1:500), p 
mTOR S-2448(Abcam, 1:500) and mTOR (Abcam, 1:1000). Goat anti-rabbit IgG-HRP and rabbit antiMouse IgG-HRP (Sigma) were used as secondary antibodies, and ECL (Amersham) reagent was used for developing. Different times were used for each protein according to the maximum activity observed. $\underline{\text { Statistical analysis }}$

Statistical analysis was performed using one-way ANOVA (GraphPad Prism 5.03 software, GraphPad Software, Inc., San Diego, CA). When the main effect was significant, the Bonferroni post hoc test was applied to determine individual differences between means. $\mathrm{P}$ values less than 0.05 were considered statistically significant.

\section{RESULTS}

\section{- S-resistin decreases insulin action in 3T3-L1 cells}

To establish the time for insulin maximal activation of the different proteins involved in the early steps on the insulin signaling pathway, we analyzed the phosphorylation of the IR, IRS-1, Akt, FoxO1a and mTOR in response to insulin stimulation at different period of time in 3T3-L1 control cells (Fig. 1). Once establish the time for insulin maximal activation of each protein in control cells, we studied the protein levels and the insulin-stimulated phosphorylation of different intermediates of the insulin signalling pathway in cells stably expressing s-resistin (3T3-L1-s-res) and resistin (3T3-L1-res), to understand the possible molecular alterations exerted by s-resistin on insulin action in these cells.

Fig. 1

First, we analyzed basal and insulin-stimulated tyrosine phosphorylation of the early proteins involved on the insulin signalling pathway like IR and IRS-1. Thus, 3T3-L1-s-res cells show reduced levels $(30 \%)$ of IR phosphorylation upon insulin treatment (Fig. 2a). This decrease was more pronounced in cells stably expressing resistin (3T3-L1-res), where IR phosphorylation dropped to the half of the values observed in insulin stimulated control cells. Also, we found that both cell types had diminished IRS-1 phosphorylation compared to 3T3-L1 control cells (Fig. 2b). In this case, s-resistin has a more significant effect, inducing a $70 \%$ decrease in IRS-1 phosphorylation compared to the $40 \%$ caused by resistin. These results confirm that s-resistin, like resistin, blocks initial steps in the pathway of insulin signalling in 3T3-L1 adipocytes.

Fig. 2 
- S-resistin decreases S473-Akt phosphorylation and mTOR activity but increases FoxO1a phosphorylation

We next studied further alterations exerted by s-resistin on the insulin signalling pathway downstream from IR and IRS-1. Specifically, we focused our attention on the phosphorylation and subsequent activation of Akt and their target mTOR. Western blot analysis demonstrated that both resistin isoforms impaired insulin-stimulated S473-Akt phosphorylation in 3T3-L1 cells, although the inhibition of resistin was more pronounced ( $75 \%$ for resistin and $50 \%$ for s-resistin, vs control, Fig. 3b). However, resistin isoforms diverged in their effects on T308-Akt phosphorylation in insulin-stimulated cells. While resistin inhibited by a 40\% T308-Akt phosphorylation, s-resistin had no effect (Fig. 3c).

Fig.3

mTOR is an Akt activated protein involved in the regulation of nutrient uptake and cell growth, among other processes [18]. Our results indicate that cells stably expressing both resistin isoforms impair insulin-stimulated mTOR phosphorylation compared to control insulin stimulated cells (22.5\% in 3T3L1-s-res and $25 \%$ in 3T3-L1-res, Fig. 4a). These results could be directly related to the decline in S473Akt phosphorylation previously observed in 3T3L1-s-res and 3T3L1-res cells (Fig. 3a).

Fig. 4

One of the target proteins inactivated by Akt phosphorylation during insulin response is the transcription factor FoxO1a. The active dephosphorylated form of FoxO1a is involved in various cellular processes such as cell cycle regulation, gluconeogenesis activation and stress response [19]. After being inactivated by Akt phosphorylation, FoxO1a is excluded from the nucleus, which leads to its degradation by the proteosome [20]. Despite the low phosphorylation levels of Akt upon insulin stimulation of cells stably expressing both resistin isoforms (Fig. 3a), surprisingly we observed a marked increase in the insulin-stimulated phosphorylation levels of FoxO1a compared with control cells, especially in 3T3-L1-sres cells (410\%, Fig. 4b).

\section{- Insulin increased SOCS-3 levels in 3T3-L1-s-res adipocytes}

The SOCS-3 protein blocks certain cytokine signals, as well as insulin and leptin pathways [21, 22]. Since previous reports demonstrated that resistin induced SOCS-3 expression in 3T3-L1 cells, inhibiting insulin action [13], we set out to analyze if s-resistin could exert a similar effect on SOCS-3. 
The results shown in Fig. 5 indicate that s-resistin increases SOCS-3 protein levels in 3T3-L1 cells after 15 minutes of incubation with insulin. However, it is noteworthy that while the 3T3-L1-res cells have elevated SOCS-3 levels even in the absence of insulin (Fig. 5a and b), in the cells stably expressing sresistin, incubation with insulin upregulated SOCS-3 expression in a time-dependent manner.

Fig. 5

- S-resistin regulates the mRNA levels of transcription factors, inflammatory cytokines and proteins involved in adipogenesis and insulin response

Table 1 summarizes the effects of resistin and s-resistin overexpression in 3T3-L1 cells on the mRNA levels of key proteins and transcription factors involved in lipid metabolism (SREBP-1c and FoxO1a), adipogenesis (mTOR), inflammation (TNF- $\alpha$ and IL-6) and insulin response (GLUT-4, SOCS3, resistin and leptin). As shown in Table 1, after 4 hours of incubation with insulin 3T3-L1-s-res cells have much higher mRNA levels of SREBP-1c, mTOR and FoxO1a. This expression pattern was also observed in the absence of insulin, suggesting that s-resistin induces lipogenesis and alters adipogenesis. In addition, the expression of GLUT-4 is significantly decreased in 3T3-L1-s-res compared to controls. These expression levels remain unchanged regardless of treatment with insulin. Besides, insulin significantly increases SOCS-3 expression in cells that express s-resistin. These results indicate that sresistin might decrease insulin sensitivity and induce leptin and insulin resistance in 3T3-L1 adipocytes.

Table 1

We next examined the expression levels of the proinflamatory cytokines TNF- $\alpha$ and IL-6. Independently of insulin, s-resistin enhanced the expression of these proinflammatory cytokines (Table 1). On the other hand, resistin expression in 3T3-L1-s-res is significantly lower than in control adipocytes. Furthermore, while in control cells resistin expression levels decrease in response to insulin, in cells expressing s-resistin this is not observed. In addition, 3T3-L1-s-res have higher leptin mRNA levels than control cells, and this difference is augmented upon insulin treatment. These results are consistent with an alteration of the adipogenic process and the induction of leptin resistance.

\section{DISCUSSION}

Resistin is an adipokine that affects insulin response in target tissues such as liver, muscle or hypothalamus, as well as on adipose tissue through a paracrine/autocrine effects. In Wistar rats, it has been described two resistin variants, resistin and s-resistin, originated by alternative splicing [11]. S- 
resistin appears as an intracellular short isoform, whose cellular functions are not clear. Several studies of different research groups indicated that the secreted form, resistin, interferes in the intracellular insulin signalling at the early stages of the pathway [6, 13, 23]. However, s-resistin effects over cell insulin signalling are still obscure. Previous results from our research group confirmed that 3T3-L1 adipocytes stably expressing s-resistin isoform, the 3T3-L1-s-res cells, exhibit a decrease in glucose transport, both basal and insulin-stimulated [8]. Here, we demonstrated that s-resistin blocks the signalling pathway of insulin since early stages through a lower activation of IR, IRS-1, Akt and mTOR, and increased mRNA and protein levels of SOCS-3.

SOCS-3 is a well-known inhibitor of insulin signalling that belongs to the suppressor of cytokine signalling (SOCS) family. It has been described that pre-treatment of 3T3-L1 adipocytes with resistin upregulates SOCS-3 expression and blocks insulin signalling pathway. In addition, 3T3-L1 adipocytes exposed to a dominant negative SOCS-3 protein prior to resistin treatment blocked the resistin-induced reduction in insulin receptor phosphorylation. Thus, resistin appears to decrease insulin signalling by increasing SOCS-3 levels [13]. SOCS-3 interacts with the insulin receptor, probably to prevent its phosphorylation, and inhibits therefore, the phosphorylation and activation of IRS1 and Akt [24].

We do not observe an increase in basal SOCS-3 mRNA levels prior to insulin treatment in 3T3L1-res cells (Table 1), probably because the increase of SOCS-3 expression described by Steppan et al. is dose-dependent and is observed when adipocytes are incubated with resistin concentrations above 10 $\mathrm{ng} / \mathrm{ml}$ [13]. However, cell clones used in this study expressed a single copy of resistin (3T3-L1-res) and sresistin (3T3-L1-s-res) transgenes, respectively [8]. Therefore, 3T3-L1-res cells have very low resistin levels in the culture medium, insufficient to induce SOCS-3 expression, although SOCS-3 protein levels remain elevated.

Nevertheless and in agreement with the work of Steppan et al. [13], our results show that insulin treatment increased SOCS-3 mRNA and protein levels in 3T3-L1-res and 3T3-L1-s-res cells, compared to control cells. These data suggest that s-resistin isoform decreases insulin signalling by up-regulating SOCS-3 through an unknown intracrine mechanism. In consequence, in 3T3-L1-s-res cells we observed a decrease in IR, IRS-1, Akt and mTOR phosphorylation as well as undetectable GLUT-4 expression levels, confirming that in adipocytes stably expressing s-resistin the insulin signal transmission was attenuated. 
In addition, we observed that both isoforms of resistin bring about an increase in the phosphorylation and consequent inactivation of FoxO1a. FoxO1a is a transcription factor involved mainly in the inhibition of hepatic glucose production and the stimulation of pancreatic $\beta$-cells proliferation by insulin. Previous studies demonstrated that Akt/PKB phosphorylates Foxo factors, leading to alteration of their transcription activity and cellular localization $[25,26]$. Despite the low levels of insulin-stimulated Akt phosphorylation exhibited by our cells, surprisingly we observed a marked increase in the phosphorylation levels of this FoxO1a, especially by s-resistin. As previously suggested, it is possible that members of mitogen-activated protein kinases (MAPK) family could phosphorylate FoxO1a [27]. More studies should be done to elucidate this point.

Furthermore, compared to control 3T3-L1 cells, 3T3-L1 cells stably expressing s-resistin increased significantly the expression levels of transcription factors involved in lipid metabolism such as SREBP1c, mTOR and FoxO1a. This would confirm that the short isoform of resistin induces alterations in adipogenesis, as FoxO1a increased the expression of various cell cycle inhibitors and Chop 10, the modulator of C/EBP, necessary for the proper development of the adipogenic process [20]. Also, it is described that the absence of FoxO1a in adipose tissue prevents insulin resistance [20]. Therefore, these high levels of FoxO1a expression confirm the evidences, described above, that both, resistin and sresistin, blocked, at least partially the insulin signalling pathway and could generate insulin resistance in adipocyte cells. On the other hand, the high levels of expression of SREBP-1c observed in the 3T3-L1-sres cell line indicate that fatty acid synthesis could be increased in these adipocytes compared to control cells. It has been described that this factor is responsible for the activation of genes that are directly involved in the lipogenic processes [28]. This result agrees with the observed increase in FAS and DGAT2 expression in these adipocytes (data not shown).

There are several studies analyzing the effect of insulin on resistin expression in adipose tissue [3, 4], in isolated adipocytes and in 3T3-L1 cells [29, 30]. Specifically, it has been reported that insulin inhibits resistin expression in 3T3-L1 adipocytes, even at sub-physiological concentrations [29]. This is consistent with our results, since we also observe a decrease in resistin expression upon incubation of 3T3-L1 cells with insulin. By contrast, in the 3T3-L1-res and 3T3-L1-s-res adipocytes, basal resistin levels are lower compared to control cells and the addition of insulin does not change resistin expression. Since resistin expression is a feature of the mature adipocyte, this decline would confirm the alterations present in both cell clones in the adipogenic process [8]. Most likely, both isoforms could regulate their 
own synthesis by increasing the expression and the release of pro-inflammatory cytokines such TNF $\alpha$ and IL-6 [8]. Given that TNF $\alpha$ impairs adipocyte differentiation, promotes insulin resistance and also acts as a potent negative regulator of resistin expression [31,32], its increased expression by s-resistin and resistin could explain the decreased insulin response and the low resistin expression observed herein.

On the other hand, previous results of our research group have shown that in isolated adipocytes [33] and in white adipose tissue [34], leptin decreases insulin response, reduces the lipogenic capacity of adipocytes and activates lipolysis, limiting thereby the size of adipose tissue. Furthermore, intracerebroventricular leptin injections in Wistar rats induced SOCS-3 expression and protein levels in adipose tissue, contributing to reduce the insulin response from this tissue [35]. Our results show that the mRNA levels of leptin are significantly increased by s-resistin and especially by the resistin isoform, concomitantly with the increase in SOCS-3 levels, indicating the important role of resistin isoforms in the development of adipocyte leptin resistance.

In summary, this work constitutes the first report on s-resistin effects on the insulin signalling pathway. Our results indicate that the s-resistin isoform, like resistin, impairs cell insulin response, possibly through a mediated increase of SOCS-3 mRNA and protein levels, as well as by inducing an inflammatory state in adipocytes. Thus, s-resistin could be also implicated in the development of adipocyte leptin resistance. Resistin is a secreted protein that may interact in a paracrine/autocrine manner with its still unidentified receptor on the adipocyte cell surface. Through unknown signal cascades, resistin increases SOCS-3 expression and impairs insulin actions. Nevertheless, s-resistin is a nonsecretable intracellular protein that could act as an intracellular sensor, by a still unknown intracrine pathway, regulating the differentiation process and cell insulin sensitivity. Further studies will be required to understand the physiological relevance of this short, non-secretable, resistin isoform.

\section{ACKOWLEDGMENTS}

MR was a recipient of a predoctoral fellowship from Universidad de Castilla-La Mancha and from BFU2012-39705-C03-01 from Ministerio de Ciencia e Innovación (Spain). This work was supported by Research Grants PI-2007/60 from Junta de Comunidades de Castilla-La Mancha (JCCM) FISCAM; DGIBFU2008-C03-02/BFI and BFU2012-39705-C03-01 from Ministerio de Ciencia e Innovación (Spain). 


\section{REFERENCES}

[1] Sisley S, Sandoval D (2011) Hypothalamic control of energy and glucose metabolism. Rev Endocr Metab Disord 12:219-33.

[2] Proenca ARG, Sertié RAL, Oliveira AC, Campaña AB, Caminhotto RO, Chimin P, Lima FB (2014) New concepts in white adipose tissue physiology. Braz J Med Biol Res 47:192-205.

[3] Steppan CM, Bailey ST, Bhat S, Brown EJ, Banerjee RR, Wrght CM, Patel HR, Ahima R, Lazar M (2001) The hormone resistin links obesity to diabetes. Nature 409:307-312.

[4] Kim K-H, Lee K, Moon YS, Sul H-S (2001) A cystein adipose tissue-specific secretory factor inhibits adipocyte differentiation. J Biol Chem 276:11252-11256.

[5] Banerjee RR, Rangwala SM, Shapiro JS, Rich AS, Rhoades B, Qi Y, Wang J, Rajala MW, Pocai A, Scherer PE, Steppan CM, Ahima RS, Obici S, Rossetti L, Lazar MA (2004) Regulation of fasted blood glucose by resistin. Science 303:1195-1198.

[6] Satoh H, Nguyen A, Miles PDG, Imamura T, Usui I, Olefski JM (2004) Adenovirus-mediated chronic "hyper-resistinemia" leads to in vivo insulin resistance in normal rats. J Clin Invest $114: 224-231$.

[7] Fu Y, Luo L, Luo N, Garvey WT (2006) Proinflammatory cytokine production and insulin sensitivity regulated by overexpression of resistin in 3T3-L1 adipocytes. Nutr Metab 19:3-28.

[8] Fernández CM, del Arco A, Gallardo N, Aguado L, Rodríguez M, Ros M, Carrascosa JM, Andrés A, Arribas C (2010) S-resistin inhibits adipocyte differentiation and increased TNF $\alpha$ expression and secretion in 3T3-L1 cells. Biochimica et Biophysica Acta 1803:1131-11741.

[9] Daquinag AC, Zhang Y, Amaya-Manzanares F, Simmons PJ, Kolonin MG (2011) An isoform of decorin is a resistin receptor on the surface of adipose progenitor cells. Cell Stem Cell 9:74-86.

[10] Sánchez-Solana B, Laborda J, Baladron V (2012) Mouse resistin modulatesadipogenesis and glucose uptake in 3T3-L1 preadipocytes through the ROR1 receptor. Mol Endocrinol 26:110127.

[11] Del Arco A, Peralta S, Carrascosa JM, Ros M, Andrés A, Arribas C (2003) Alternative splicing generates a novel non-secretable resistin isoform in Wistar rats. FEBS Letters 555:243-249. 
[12] Fernández CM, Moltó E, Gallardo N, del Arco A, Martínez C, Andrés A, Ros M, Carrascosa JM, Arribas C (2009) The expression of rat resistin isoforms is differentially regulated in visceral adipose tissues: effects of aging and food restriction. Metabolism Clinical and Experimental 58:204-211.

[13] Steppan CM, Wang J, Whiteman EL, Birnbaum MJ, Lazar MA (2005) Activation of SOCS-3 by resistin. Mol Cell Biol 25:1569-75.

[14] Muse ED, Obici S, Bhanot S, Monia BP, McKay RA, Rajala MW, Scherer PE, Rossetti L (2004) Role of resistin in diet-induced hepatic insulin resistance. J Clin Invest 114:232-239.

[15] Frost SC, Lane MD (1985) Evidence for the involvement of vicinal sulfhydryl groups in insulinactivated hexose transport by 3T3-L1 adipocytes. J Biol Chem 260:1646-2652.

[16] Peralta S, Carrascosa JM, Gallardo N, Ros M, Arribas C (2002) Ageing increases SOCS-3 expression in rat hypotalamus: effects of food restriction. Biochem Biophys Res Comm 296:425428.

[17] Livak K, Schmittgen T (2001) Analysis of relative gene expression data using real-time quantitative PCR and the 2(-delta delta C (T) method. Methods 25:402-408.

[18] Laplante M, Sabatini DM (2012) mTOR signalling in growth control and disease. Cell 149:274-293.

[19] Ronnebaum SM, Patterson C (2010) The FoxO family in cardiac function and dysfunction. Annu Rev Physiol 72:81-94.

[20] Nakae J, Kitamura T, Kitamura Y, Biggs WH, Arden KC, Accili D (2003) The Forkhead transcription factor Foxo1 regulates adipocyte differentiation. Dev Cell 4:119-129.

[21] Krebs DL, Hilton DJ (2001) SOCS proteins: negative regulators of cytokine signalling. Stem Cells 19:378-87.

[22] Morton GJ, Schwatz MW (2011) Leptin and the central nervous system control of glucose metabolism. Physiol Rev 91:389-411.

[23] Palanivel R, Maida A, Liu Y, Sweeney G (2006) Regulation of insulin signalling, glucose uptake and metabolism in rat skeletal muscle cells upon prolonged exposure to resistin. Diabetologia 49:183-90.

[24] Barnes KM, Miner JL (2009) Role of resistin in insulin sensitivity in rodents and humans. Curr Protein Pept Sci 10:96-107. 
[25] Tran H, Brunet A, Griffith EC, Greenberg ME (2003) The many forks in FOXO's road. Sci STKE 172:RE5.

[26] Matsuzaki H, Daitoku H, Hatta M, Tanaka K, Fukamizu A (2003) Insulin-induced phosphorylation of FKHR (Foxo1) targets to proteasomal degradation. Proc Natl Acad Sci U S A 100:11285-90.

[27] Asada S, Daitoku H, Matsuzaki H, Saito T, Sudo T et al. (2007) Mitogen-activated protein kinases, Erk and p38, phosphorylate and regulate Foxo 1. Cellular signalling 19: 519-527.

[28] Nadeau KJ, Letiner JW, Gurerich I, Draznin B (2004) Insulin regulation of sterol regulatory element-binding protein-1 expression in L-6 muscle cells and 3T3-L1 adipocytes. J Biol Chem 279:34380-87.

[29] Haugen F, JØrgensen A, Drevon CA, Trayhurn P (2001) Inhibition by insulin of resistin gene expression in 3T3-L1 adipocytes. FEBS Lett 507:105-108.

[30] Liu F, Fan HQ, Qiu J, Wang B, Zhang M, Gu N, Zhang CM, Fei L, Pan XQ, Guo M, Chen RH, Guo XR (2008) A paradox: insulin inhibits expression and secretion of resistin which induces insulin resistance. World J Gastroenterol 14:95-100.

[31] Shojima N, Sakoda H, Ogihara T, Fujishiro M, Katagiri H, Anai M, Onishi Y, Ono H, Inukai K, Abe M, Fukushima Y, Kikuchi M, Oka Y, Asano T (2002) Humoral regulation of resistin expression in 3T3-L1 and mouse adipose cells. Diabetes 51:1737-44.

[32] Li Y, Totsune K, Takeda K, Furuyama K, Shibahara S, Takahashi K (2003) Differential expression of adrenomedullin and resistin in 3T3-L1 adipocytes treated with tumor necrosis factoralpha. Eur J Endocrinol 149:231-8.

[33] Pérez D, Fernández-Galaz C, Fernández-Agulló T, Arribas C, Andrés A, Ros M, Carrascosa JM (2004) Leptin impairs insulin signaling in rat adipocytes. Diabetes 53:347-353.

[34] Gallardo N, Bonzón-Kulichenko E, Fernández-Agulló T, Moltó E, Gómez-Alonso S, Blanco P, Carrascosa JM, Ros M, Andrés A (2007) Tissue-Specific Effects of Central Leptin on the Expression of Genes Involved in Lipid Metabolism in Liver and White Adipose Tissue. Endocrinology 148:5604-10.

[35] Bonzón-Kulinchenko E, Fernández-Agulló T, Moltó E, Serrano R, Fernández A, Ros M, Carrascosa JM, Arribas C, Martínez C, Andrés A, Gallardo N (2011) Regulation of insulin- 
stimulated glucose uptake in rat White adipose tissue upon chronic central leptin infusion: effects on adiposity. Endocrinology 152:1366-77.

\section{FIGURE LEGENDS}

Fig. 1.

Fig. 2. Effect of resistin and s-resistin on the activity of IR and IRS-1 in 3T3-L1 cells stimulated with insulin. Control 3T3-L1 cells, as well as cells stably expressing resistin (3T3-L1-res) and the short resistin isoform (3T3-L1-s-res) were treated for $5 \mathrm{~min}$ (for IR) or $10 \mathrm{~min}$ (for IRS-1) with $100 \mathrm{nM}$ insulin. Representative western blots show total (T IR $\beta$, T IRS-1) and phosphorylated (P Y1146-IR $\beta$, P Y632IRS-1) levels of IR (A) and IRS-1 (B). Densitometry analysis of the degree of phosphorylation of IR $\beta$ (C) and IRS-1 (D) upon stimulation with insulin is presented. Values are the means \pm SEM; $\mathrm{n}=3$ independent experiments per group of cells. Values that do not share the same superscript letter are significantly different $(\mathrm{p} \leq 0.05)$.

Fig. 3. Effect of resistin and s-resistin on the activity of Akt in 3T3-L1 cells stimulated with insulin. Control 3T3-L1 cells, as well as cells stably expressing resistin (3T3-L1-res) and the short resistin isoform (3T3-L1-s-res) were treated for $15 \mathrm{~min}$ with $100 \mathrm{nM}$ insulin. Representative western blots (A) show total (T Akt) and phosphorylated S473-Akt (P S473-Akt), T308-Akt (P T308-Akt) levels of Akt. Densitometry analysis of the degree of phosphorylation on S473-Akt (B) or T308-Akt (C) upon stimulation with insulin is presented. Values are the means $\pm S E M ; n=3$ independent experiments per group of cells. Values that do not share the same superscript letter are significantly different $(\mathrm{p} \leq 0.05)$.

Fig. 4. Effect of resistin and s-resistin on the activity of mTOR and FoxO1a in 3T3-L1 cells stimulated with insulin. Control 3T3-L1 cells, as well as cells stably expressing resistin (3T3-L1-res) and the short resistin isoform (3T3-L1-s-res) were treated for $30 \mathrm{~min}$ with $100 \mathrm{nM}$ insulin. Representative western blots show total (T mTOR and T FoxO1a) and phosphorylated (P S2448-mTOR and P S319FoxO1a) levels of mTOR (A) and FoxO1a (B). Densitometry analysis of the degree of phosphorylation of mTOR (C) or FoxO1a (D) upon stimulation with insulin is presented. Values are the means \pm SEM; $\mathrm{n}=3$ independent experiments per group of cells. Values that do not share the same superscript letter are significantly different $(\mathrm{p} \leq 0.05)$. 
Fig. 5. Effect of resistin and s-resistin on SOCS-3 protein levels in 3T3-L1 cells stimulated with insulin. Control 3T3-L1 cells, as well as cells stably expressing resistin (3T3-L1-res) and the short resistin isoform (3T3-L1-s-res) were treated for $15 \mathrm{~min}$ with $100 \mathrm{nM}$ insulin. Representative western blots (A) show SOCS-3 total levels. Densitometry analysis (B) of the protein level of SOCS-3 upon stimulation with insulin is presented. Values are the means $\pm \mathrm{SEM} ; \mathrm{n}=3$ independent experiments per group of cells. Values that do not share the same superscript letter are significantly different $(\mathrm{p} \leq 0.05)$. 


\begin{tabular}{|c|c|c|c|c|c|c|}
\hline \multirow{2}{*}{ Gene } & \multicolumn{2}{|c|}{ 3T3-L1 } & \multicolumn{2}{|c|}{ 3T3-L1-res } & \multicolumn{2}{|c|}{ 3T3-L1-s-res } \\
\hline & $(-)$ Ins & $(+) \operatorname{lns}$ & $(-)$ Ins & $(+) \operatorname{lns}$ & $(-)$ Ins & $(+)$ Ins \\
\hline \multicolumn{7}{|c|}{ Transcription factors related to lipid metabolism and adipogenesis } \\
\hline SREBP-1C & $1.00 \pm 0.07^{a}$ & $1.2 \pm 0.3^{a}$ & $0.60 \pm 0.04^{b}$ & $1.83 \pm 0.03^{c}$ & $3.4 \pm 0.3^{d}$ & $2.3 \pm 0.1^{\mathrm{e}}$ \\
\hline Fox01a & $1.0 \pm 0.2^{a}$ & $0.95 \pm 0.06^{a}$ & $1.7 \pm 0.1^{b}$ & $2.4 \pm 0.1^{c}$ & $1.9 \pm 0.1^{b}$ & $2.5 \pm 0.2^{c}$ \\
\hline mTOR & $1.00 \pm 0.08^{a}$ & $1.25 \pm 0.02^{\mathrm{a}}$ & $1.9 \pm 0.5^{b}$ & $3.3 \pm 0.3^{c}$ & $4.0 \pm 0.2^{d}$ & $4.0 \pm 0.2^{d}$ \\
\hline \multicolumn{7}{|c|}{ Inflammatory citokines } \\
\hline $\mathrm{TNF}_{\boldsymbol{\alpha}}$ & $1.00 \pm 0.04^{a}$ & $0.59 \pm 0.05^{b}$ & $2.16 \pm 0.01^{c}$ & $1.51 \pm 0.01^{d}$ & $6.5 \pm 0.2^{e}$ & $4.8 \pm 0.2^{f}$ \\
\hline IL-6 & $1.0 \pm 0.2^{a}$ & $1.02 \pm 0.09^{a}$ & $4.33 \pm 0.04^{b}$ & $5.5 \pm 0.6^{c}$ & $1.7 \pm 0.3^{d}$ & $4.6 \pm 0.5^{b, c}$ \\
\hline \multicolumn{7}{|c|}{ Insulin signal and adipokines } \\
\hline GLUT-4 & $1.00 \pm 0.02^{\mathrm{a}}$ & $1.3 \pm 0.2^{b}$ & $0.60 \pm 0.04^{c}$ & $2.0 \pm 0.1^{d}$ & $0.05 \pm 0.02^{e}$ & $0.04 \pm 0.01^{e}$ \\
\hline socs-3 & $1.00 \pm 0.01^{a}$ & $0.05 \pm 0.01^{b}$ & $0.90 \pm 0.06^{a}$ & $2.98 \pm 0.01^{c}$ & $0.3 \pm 0.1^{d}$ & $1.71 \pm 0.01^{e}$ \\
\hline Resistin & $1.0 \pm 0.1^{a}$ & $0.52 \pm 0.02^{b}$ & $0.05 \pm 0.02^{c}$ & $0.16 \pm 0.09 c$ & $0.6 \pm 0.1^{b}$ & $0.39 \pm 0.02^{b}$ \\
\hline Leptin & $1.0 \pm 0.2^{a}$ & $1.38 \pm 0.09^{a}$ & $26 \pm 7^{b}$ & $56 \pm 1^{c}$ & $5 \pm 1^{d}$ & $14.5 \pm 0.9^{e}$ \\
\hline
\end{tabular}

Table 1. Effect of the rat s-resistin isoform on mRNA levels of inflammatory cytokines, different transcription factors related to lipid metabolism, as well as on other proteins involved in adipogenesis or adipocyte insulin response. The expression was determinated by qPCR using SYBRgreen or Taqman depending on the gen (see Materials and Methods). 18S rRNA was used as endogenous control. Values are the means \pm SEM; $n=3$ independent experiments per group, each sample made in triplicate. Values that do not share the same superscript letter are significantly different $(\mathrm{p}<0.05)$. (- Ins), without insulin; (+ Ins), with insulin. 
A)
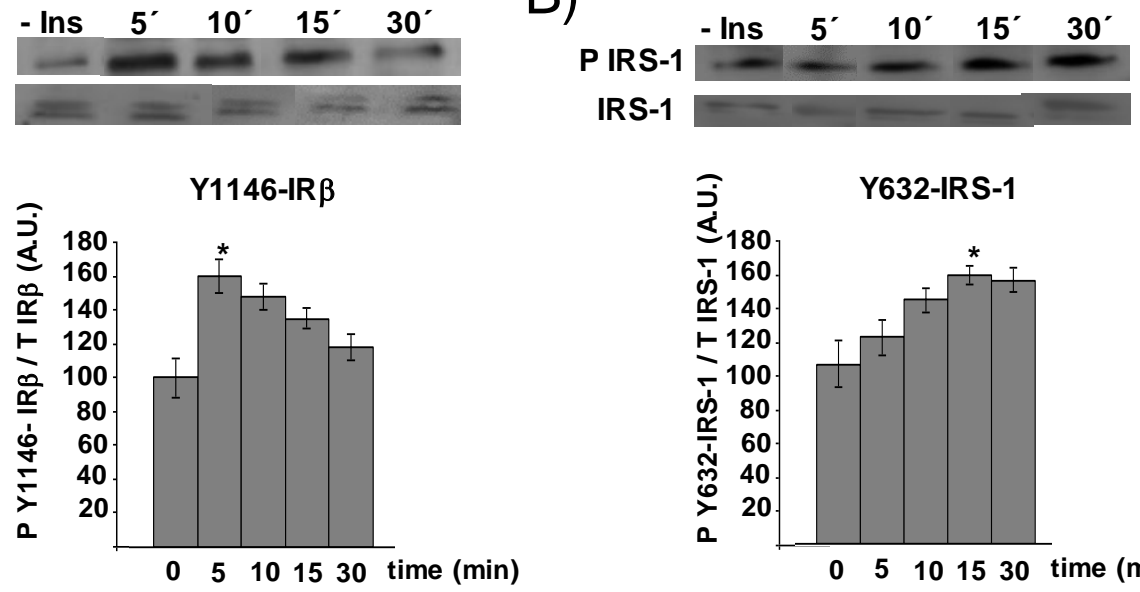

D)
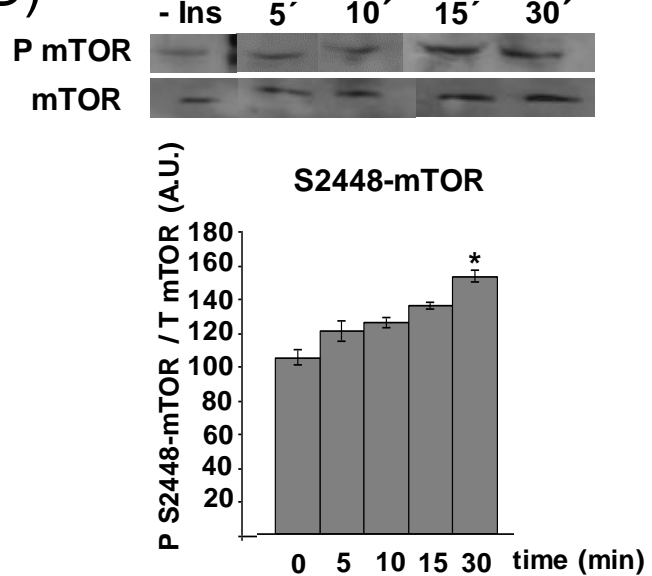

E)

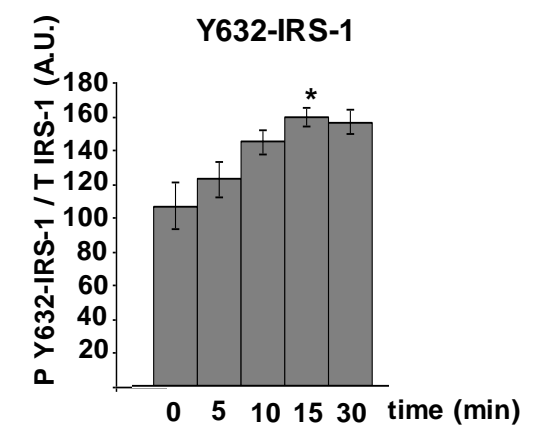

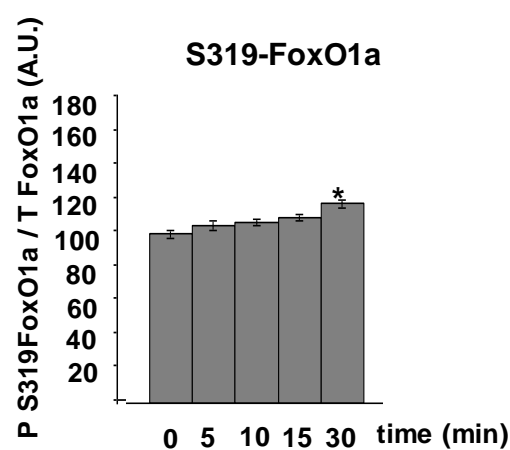

C)

$\begin{array}{lllll}- \text { Ins } & 5^{\prime} & 10^{\prime} & 15^{\prime} & 30^{\prime}\end{array}$

P S Akt

P T Akt

Akt

S473-Akt

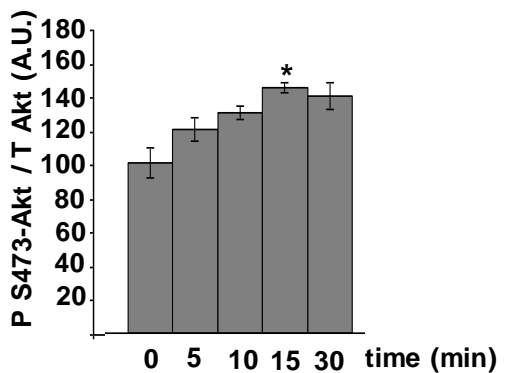

T308-Akt

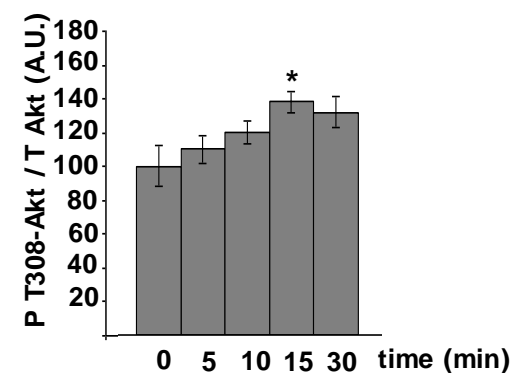

F)

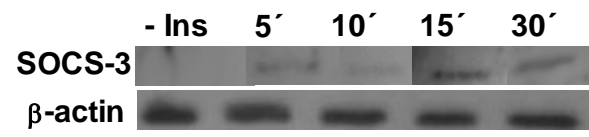

SOCS-3

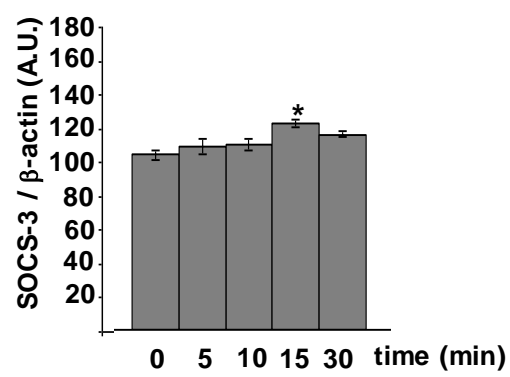

Figure 1. Time-course phosphorylation of different intermediates of the insulin signalling pathway in response to insulin in 3T3-L1 control cells. The asterisks indicate the time for the maximal activation of each protein. A)-E) Phosphorylation levels of each protein F) Total levels. 

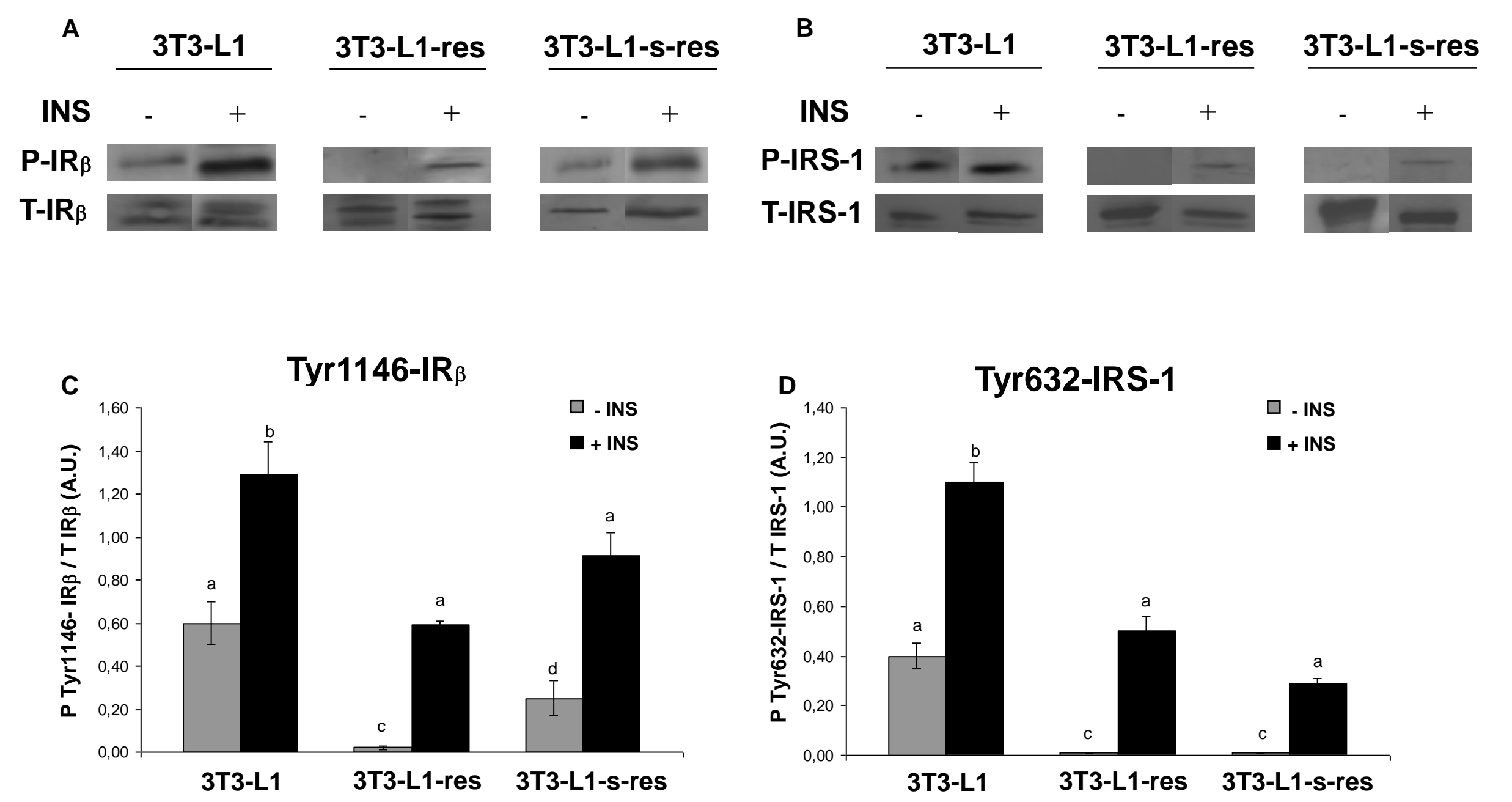

Figure 2 Effect of resistin and s-resistin on the activity of the insulin receptor (IR) and insulin receptor substrate-1 (IRS-1) in 3T3-L1 cells stimulated with insulin. Control3 3-L1 cells, as well as cells stably expressing resistin (3T3-L1-res) and the short resistin isoform (3T3-L1-s-res) were treated for 5 min (for IR) or 15 min (for IRS-1) with $100 \mathrm{nM}$ insulin. Representative western blots show total (T IR $\beta$, T IRS-1) and phosphorylated (P Tyr1146-IR $\beta$,P Tyr632-IRS-1) levels of IR (A) and IRS-1 (B). Densitometry analysis of the degree of phosphorylation of $\operatorname{IR} \beta(C)$ and IRS-1 (D) upon stimulation with insulin is presented. Values are the means $\pm S E M ; n=3$ independent experiments per group of cells.

Values that do not share the same superscript letter are significantly different $(p \leq 0.05)$. 

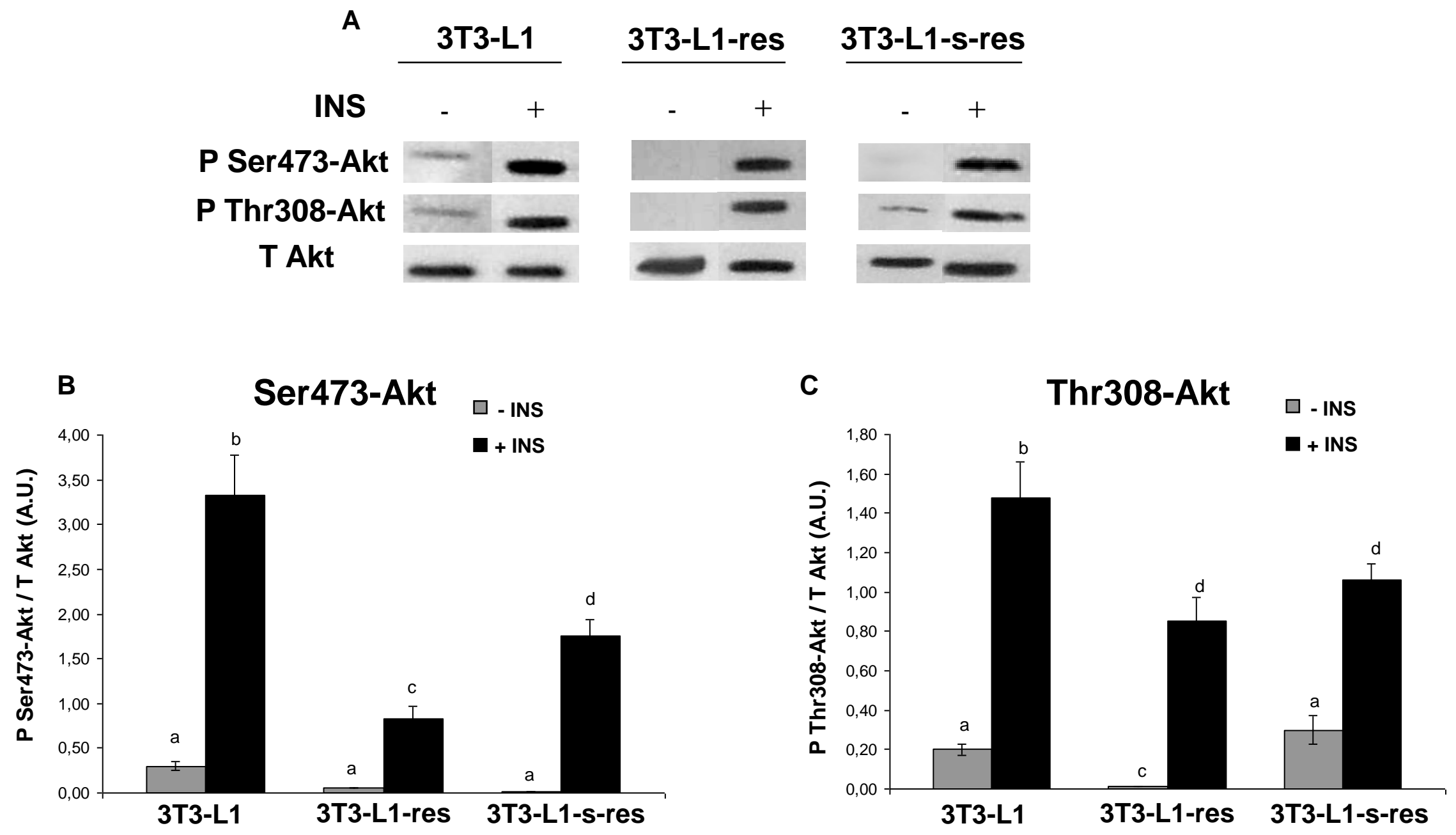

Figure 3 ffect of resistin and s-resistin on the activity of protein quinase B (Akt) in 3T3-L1 cells stimulated with insulin.

Control 3T3-L1 cells, as well as cells stably expressing resistin (3T3-L1-res) and the short resistin isoform (3T3-L1-S-res)

were treated for $15 \mathrm{~min}$ with $100 \mathrm{nM}$ insulin. Representative western blots (A) show total (T Akt) and phosphorylated Ser473-Akt (P 473-Ser Akt),

Thr308-Akt (P Thr308-Akt) levels of Akt. Densitometry analysis of the degree of phosphorylation on Ser473-Akt (B) or Thr308-Akt (C) upon stimulation with insulin is presented. Values are the means $\pm S E M ; n=3$ independent experiments per group of cells.

Values that do not share the same superscript letter are significantly different $(p \leq 0.05)$. 


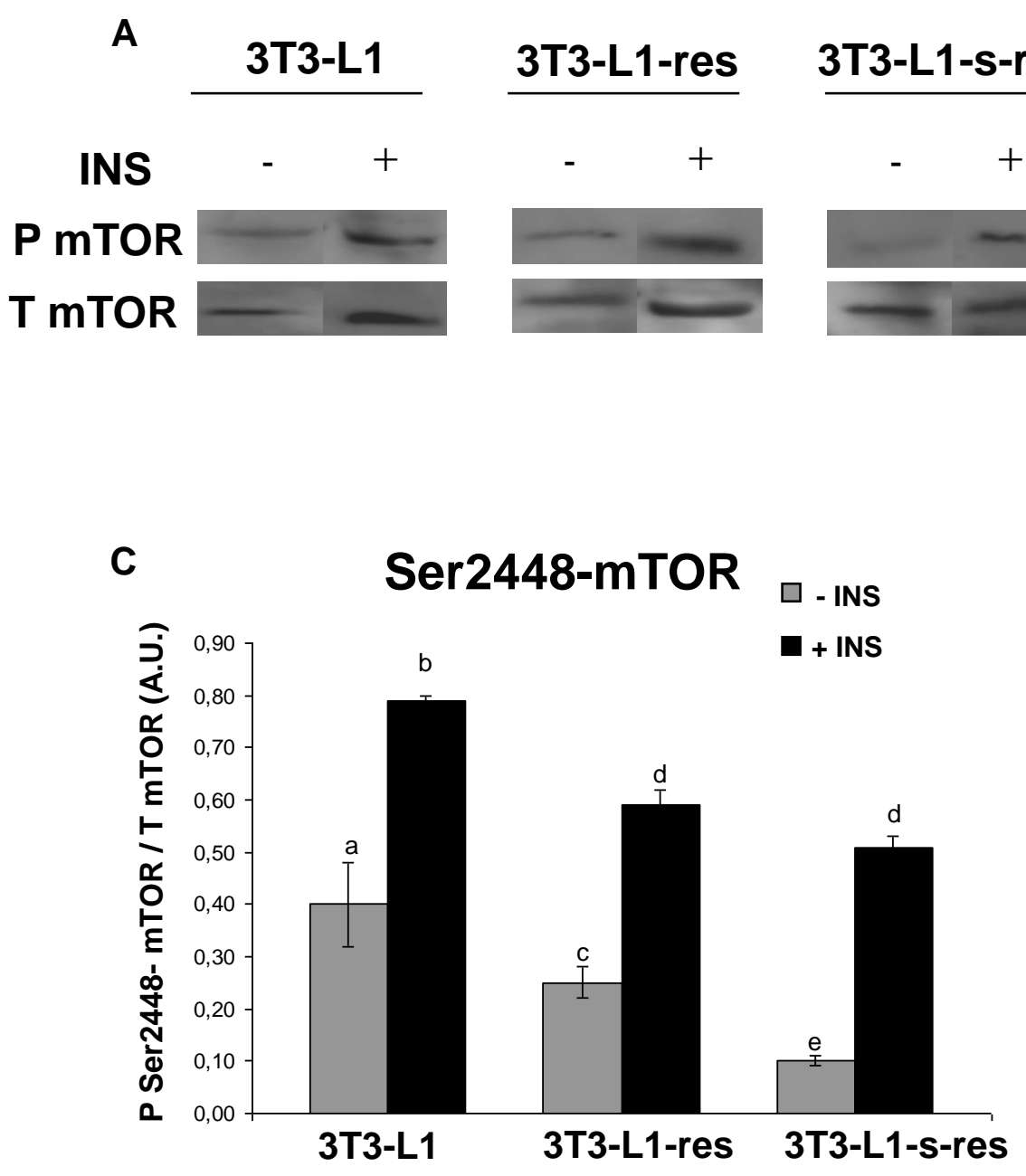

B

3T3-L1

3T3-L1-res

3T3-L1-s-res

INS
P Fox01a
T Fox01a

$+$

$+$
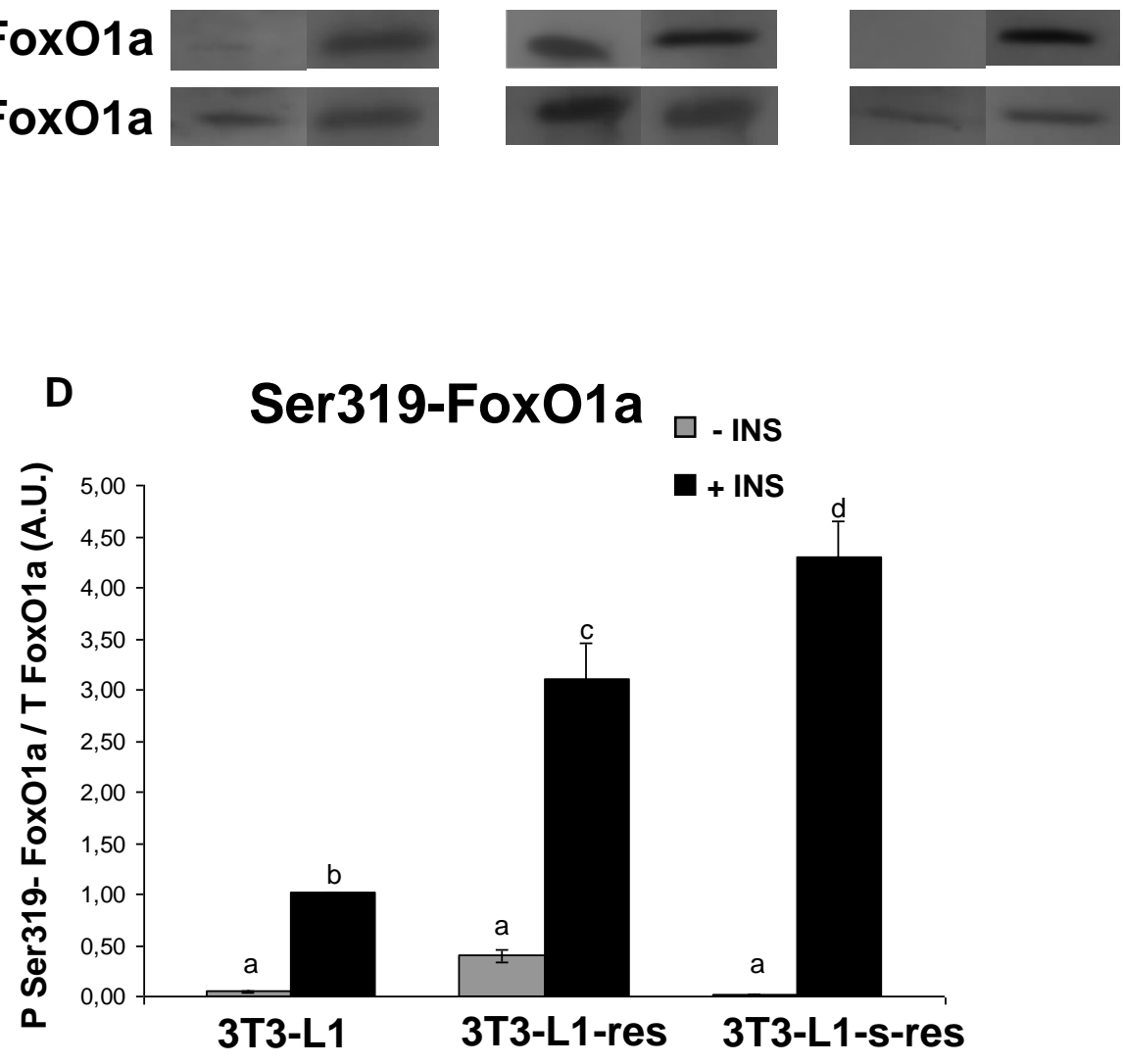

Figure 4 Effect of resistin and s-resistin on the activity of mTOR and FoxO1a in 3T3-L1 cells stimulated with insulin.

Contro 3T3-L1 cells, as well as cells stably expressing resistin (3T3-L1-res) and the short resistin isoform (3T3-L1-S-res)

were treated for $30 \mathrm{~min}$ with $100 \mathrm{nM}$ insulin. Representative western blots show total (T mTOR and T FoxO1a) and phosphorylated (P Ser2448-mTOR and P Ser319-FoxO1a) levels of mTOR (A) and FoxO1a (B). Densitometry analysis of the degree of phosphorylation of mTOR (C) or FoxO1a (D) upon stimulation with insulin is presented.

Values are the means $\pm S E M ; n=3$ independent experiments per group of cells. Values that do not share the same superscript letter are significantly different ( $p \leq 0.05)$. 


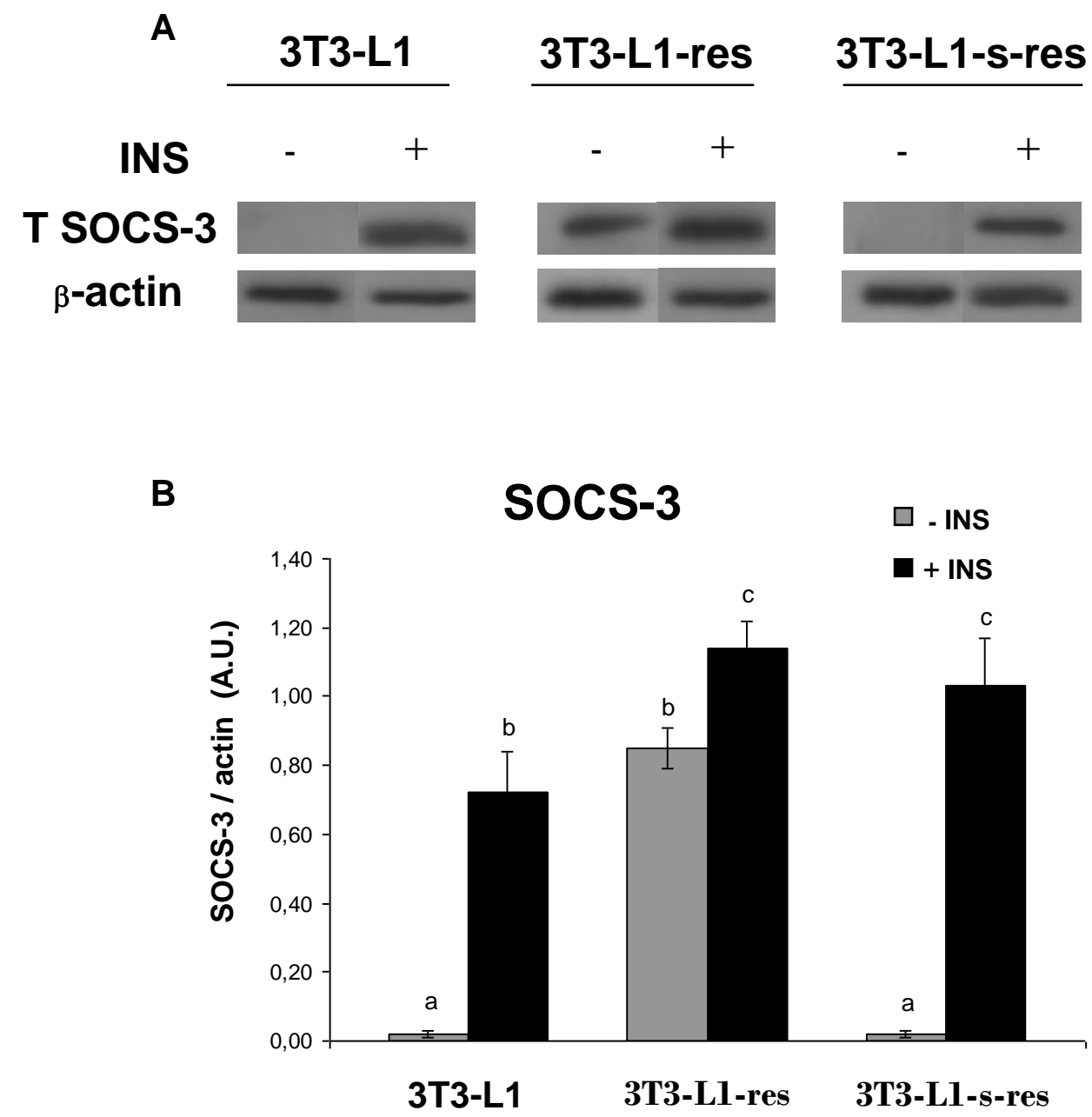

Figure 5 Effect of resistin and s-resistin on SOCS-3 protein levels in 3T3-L1 cells stimulated with insulin.

Control 3T3-L1 cells, as well as cells stably expressing resistin (3T3-L1-res) and the short resistin isoform (3T3-L1-s-res) were treated for 15 min with $100 \mathrm{nM}$ insulin. Representative western blots (A) show SOCS-3 total levels.

Densitometry analysis (B) of the protein level of SOCS-3 upon stimulation with insulin is presented.

Values are the means $\pm S E M ; n=3$ independent experiments per group of cells. Values that do not share the same superscript letter are significantly different ( $p \leq 0.05$ ). 